\title{
Safety and Efficacy of Neuronavigation via Middle Frontal Gyrus Endoport-assisted Endoscopic Resection for Thalamic Lesions
}

\section{Shuang Liu}

Fudan University

\section{Tao Xie}

Fudan University

\section{Yeh Yu Yang}

Fudan University

Chen Li

Fudan University

Tengfei Liu

Fudan University

Chongjing Sun

Fudan University

\section{Silin Wu}

Fudan University

\section{Liangliang Yang}

Fudan University

\section{Zeyang Li}

Fudan University

\section{Fan $\mathrm{Hu}$}

Fudan University

Xiaobiao Zhang ( $\square$ xiaobiao_zhang@163.com )

Fudan University https://orcid.org/0000-0003-3970-1106

\section{Research Article}

Keywords: thalamic lesions, endoport, endoscopy, neuronavigation, prognosis

Posted Date: October 18th, 2021

DOI: https://doi.org/10.21203/rs.3.rs-974574/v1 
License: (c) (i) This work is licensed under a Creative Commons Attribution 4.0 International License. Read Full License 


\section{Abstract}

\section{Background}

Surgery for thalamic lesions is generally challenging because they are deep-seated and surrounded by vital neurovascular structures. Whether neuronavigation via middle frontal gyrus endoport-assisted endoscopic resection for thalamic lesions is safe and effective remains to be further evaluated.

Materials and Methods

Twelve patients treated surgically were retrospectively reviewed using the neuronavigation endoportassisted endoscopy between January 2016 and April 2021 at Zhongshan Hospital of Fudan University. Preoperative and tumor-related variables, as well as postoperative outcomes, were also collected.

Results

All lesions located in the medial part of the thalamus, and some of them expanded forward, downward, or backward. The median size of lesions was $29 \mathrm{~mm}$ (range 16-56 mm). The final pathology results showed 4 cases of benign lesions, 4 cases of low-grade glioma, and 4 cases of glioblastoma. All of the cases with benign lesions and low-grade glioma (100\%) achieved GTR, while 3/4 (75\%) of cases with glioblastoma achieved NTR, and 1 (25\%) case obtained STR. None of the patients in this study have postoperative seizures. In the benign lesions and low-grade glioma group, worse Karnofsky performance status scores at discharge were $25 \%$, and all achieved long-term postoperative survival. For patients with glioblastoma, $3 / 4$ cases had worse Karnofsky performance status scores at discharge, and died within 6 months.

Conclusion

Combining the advantages of neuronavigation, endoscopy, and endoport techniques via the middle frontal gyrus approach can safely and effectively remove benign lesions and low-grade glioma in the medial part of the thalamus.

\section{Introduction}

Thalamic lesions are rare, comprising about $1-5 \%$ of all brain tumors[8]. The clinical presentations mainly include increased intracranial pressure, obstructive hydrocephalus, motor deficits, sensory dysfunctions, and visual problems [6, 21, 27]. Since these lesions are deep-seated and surrounded by vital neurovascular structures, surgery has been generally considered challenging $[2,3,5]$. Therefore, a conservative approach was preferred over a radical resection $[2,5,25]$. However, recent advances in neuroimaging and surgical techniques have made surgical resection of thalamic lesions feasible, and the patient's prognosis has been significantly improved compared with palliative care [7]. 
Clinically, fully exposing the central thalamic lesions while minimizing damage to the surrounding brain structure is the key to the surgery. Using the neuroendoscopy technology can improve the illumination of the surgical field, better define anatomical details, and provide a closer view to observe the tumor-tissue interface, thereby increasing the precision of the operation $[9,12,13]$. However, the endoscopic approach also has limitations, including restricted movements of the instruments, a visual field frequently obscured by intraoperative bleeding, and the inability to use a two-handed technique [17]. More importantly, to access deep-seated lesions, transcortical approaches require a corticectomy and some degree of dissection of the overlying white matter[20]. The endoport is a recent development for the treatment of deep-seated intracranial lesions[10]. It allows for smaller craniotomies, causes a minor violation of the cortex and disturbance of the subcortical white matter fiber tracts compared to traditional transcortical approaches[20]. Under neuronavigation, the combination of these two technologies has recently been used in surgical procedures for deep brain and intraventricular lesions [4].

The study retrospectively evaluated the safety and efficacy of neuronavigation endoport-assisted endoscopic resection for thalamic lesions in our center. We present that this system can reduce brain damage, maintain a safe corridor, enhance the accessibility of endoscopes, and finally lead to complete lesion removal.

\section{Materials And Methods}

\section{Study Population}

This study retrospectively evaluated patient data from our electronic medical records; Patient identities were anonymized before analysis. The entire study complied with the ethical guidelines of the 1975 Declaration of Helsinki (as revised in Brazil in 2013). The Zhongshan Hospital Research Ethics Committee approved the study protocol. Director's consent for publication was granted as well.

Adult patients diagnosed with thalamic lesions and received surgery for tumor resection from January 2016 to April 2021 at Zhongshan Hospital were identified. Patients who also met the following criteria were enrolled: 1. Preoperative imaging data suggested the thalamus lesion; 2. Neuronavigation via middle frontal gyrus endoport-assisted endoscopic resection; 3 . The main body of the lesion was confirmed intraoperatively in the thalamus. 4. And postoperative pathology supported the clinical diagnosis. Finally, a total of 12 patients were enrolled in the current study.

All patients underwent a neurological examination at admission, immediately after surgery, at discharge, and three months postoperatively. We collected their variables, including age, sex, clinical symptoms, imaging data, Karnofsky performance status (KPS) score before and after surgical treatment, tumor size, tumor location, pathology, postoperative outcomes, and other variables. The tumor location was described based on the classification from Rangel-Castilla and Spetzler[22]. Classification of the thalamus into six different regions according to the anatomical structure and the most suitable surgical method. The Follow-up interval was depending on the histopathology of the lesion. 


\section{Endoscopic Surgery Procedure}

All patients underwent contrast-enhanced cranial magnetic resonance imaging (MRI) and other routine preoperative examination. In addition, magnetic resonance venography, diffusion tensor imaging were also added, if necessary. The neurosurgical team conducted preoperative discussions and formulated the surgical plan according to the examination results, patient's physical condition, and clinical symptoms. The main variables that determined the choice of surgical approach included: age, size, and location of the lesion; suspected histopathology; a relationship of the lesion to the ventricular system and corpus callosum; lesion relationship with the deep venous system and the tentorial angle; and presence of disturbances in the circulation of cerebrospinal fluid.

Three-dimensional magnetization-prepared rapid gradient-echo MRI was performed and registered to the neuronavigation system (Excelim-04 Image-guided System, Fudan Digital Medical Company, Shanghai, China) preoperatively.

\section{Surgical Techniques (Endoscopic Transcortical Transventricular Approach)}

After general endotracheal anesthesia, patients were placed into a three-point cranial fixation with the Mayfield skull clamp system (Integra NeuroCare, San Diego, CA, USA) to maintain a supine position with the upper body elevated 30 degrees. The tumor laterality mainly determined the side of the surgical approach but also depended on the surgeon's preference. According to the precise navigation guidance, a straight incision about $5 \mathrm{~cm}$ long was located anterior to the middle frontal gyrus. Then, a small circular craniotomy bone flap with approximately $3 \mathrm{~cm}$ diameter was made to allow adequate room for the endoport device (Figure $1 \mathrm{~A}$ ), navigated again to verify the puncture direction and incised from the cortex to the lateral ventricle in the hypovascular area. The BrainPath endoport system (NICO, Indianapolis, IN, USA) was utilized for all patients. We chose the appropriate endoport system (generally $21 \mathrm{~mm} / 7 \mathrm{~cm}$ or $28 \mathrm{~mm} / 7 \mathrm{~cm}$ model) to expand the cortex and advance along the planned trajectory until the lateral ventricle (Figure 1B). Snake-shaped automatic retractor fixed the outer sheath of the endoport. After removing the obturator, the 0-degree endoscope (4-mm diameter, 18-cm length, 0-degree lenses; Karl Storz $\mathrm{GmbH} \&$ Co., Tuttlingen, Germany) is introduced into the surgical field and attached to the pneumatic selfretaining holding device (Figure 1C). Both the outer sheath and the endoscope can move dynamically in any plane during the procedure. After identifying the vital structures in the lateral ventricle and tumor protruding into the ventricle, we separated the tumor from the surrounding tissue (Figure 2). When the tumor is too large to achieve en bloc resection, intratumoral decompression followed by piecemeal total resection was adopted. (Figure 3). When necessary, the angle lens was used to view the anatomic details and tumor boundary. Then, the endoscope can remove the intraventricular clot and confirm no residual tumor and hematoma. Finally, we closed the dura in a watertight fashion with reserved fascia. In addition, the craniotomy bone flap was affixed back to the skull with a titanium plating system, and standard layered closure was performed.

CT scan was performed immediately after the operation for all of the patients. Then they were admitted to the neurosurgery intensive care unit to observe for 24 hours. After the patient's condition stabilized, 
they were transferred to the general ward to monitor neurological symptoms and receive supportive treatment. We routinely used the Sodium Valproate up to 2 weeks after surgery to prevent seizures (Intravenous infusion of Sodium Valproate was applied during the perioperative period, and then it was changed to oral after the diet was restored (Monitor blood concentration during treatment). We recommend that they undergo corresponding radiotherapy and chemotherapy based on the histological characteristics of the tumors. The degree of tumor resection was based on operation notes compared to preoperative and the first postoperative CT or MRI studies. The extent of resection was defined as grosstotal resection (GTR), near-total resection (NTR), and subtotal resection (STR), based on both the surgical findings and postoperative iamges.

\section{Results}

\section{Patient characteristics}

Twelve patients were referred to our institution for neuronavigation endoport-assisted endoscopic resection for thalamic lesions (Table 1 ). The preoperative clinical symptoms of the patient mainly manifested as headache or dizziness (58.3\%) and hemiparesis (25.0\%). In addition, two patients (16.7\%) showed ptosis of the upper lid and diplopia. A total of 5 patients received preoperative treatment, including 2 received external ventricular drainage and third ventriculostomy individually, and 3 received medical treatment to alleviate edema. All of them are located in the medial part of the thalamus (region 2), and some of them expanded forward, downward, or backward. The median size of tumors is $29 \mathrm{~mm}$. The final pathology results suggested that 1 case was cavernous malformation, 3 were pilocytic astrocytoma, 4 were low-grade glioma, and 4 were glioblastoma.

\section{The extent of resection and clinical outcome}

We combined cases of cavernous malformation, pilocytic astrocytoma, and low-grade gliomas into one group and compared the resection results with cases of glioblastoma (Table 2). All of the cases with benign lesions and low-grade glioma (100\%) achieved GTR (Figure 4), while $3 / 4$ cases (75\%) with glioblastoma achieved NTR, and 1 case (25\%) obtained STR (Figure 5). In the glioblastoma group, all patients experienced clinical symptoms unrelieved or new-onset after surgery. Moreover, $3 / 4$ of patients (75\%) had worse KPS scores at discharge and present postoperative complications, including severe pneumonia and deep venous thrombosis. In the benign lesions and low-grade gliomas group, one case (25\%) developed new-onset clinical symptoms (uncoordinated limb movement), and 2 cases had worse KPS scores. During the operation, 2 cases underwent endoscopic third ventriculostomy and fistulization of the pellucid septum to prevent hydrocephalus. In addition, $3 / 8$ cases $(37.5 \%)$ in this group present postoperative complications but are not severe, including serum electrolyte disorder, transient voiding dysfunction, and subdural hematoma that does not require surgical intervention. None of the patients in this study have postoperative seizures. The median ICU days were 4.5 days (range 1-7 days) in the glioblastoma group, significantly longer than benign lesions and the low-grade glioma group (1 day).

\section{Survival outcomes}


For patients with benign lesions, all achieved long-term postoperative survival. Four patients with lowgrade glioma also obtained satisfying outcomes after receiving adjuvant radiotherapy. By the end of the follow-up, none of these eight patients died (Figure 6). For patients with glioblastoma, 2 of 4 patients lost the opportunity to receive adjuvant treatment due to severe postoperative complications. They survived for 2 and 3 months, respectively. For the remaining 2 cases received postoperative adjuvant chemotherapy and radiotherapy, one had tumor recurrence within 6 months after surgery and died subsequently, the other case had no signs of tumor recurrence after 7 months follow up.

\section{Discussion}

The basic principle of thalamic lesion surgery is to fully expose the target area and minimize the risk of damaging the surrounding anatomical structures [1]. Our study showed that neuronavigation via middle frontal gyrus endoport-assisted endoscopic surgery could increase the range of deep exposure and reduce the risk of damaging the adjacent neurovascular, thereby removing the thalamic lesions safely and effectively. Therefore, through detailed preoperative imaging evaluation, selecting appropriate patients with thalamic lesions to undergo the surgical procedure can achieve the purpose of alleviating symptoms, removing the tumor to the greatest extent, and confirming the pathological results.

The deep location of the thalamus poses a massive challenge to surgeons, as most of its surface is covered by the internal capsule laterally and by the hypothalamus and the mesencephalon anteroinferior. However, part of the thalamus surface is also between the lateral ventricles, forming the third ventricle lateral walls. The ventricular system provides sufficient in-depth observation space for endoscopic hypothalamic tumor resection with minimal damage to brain parenchyma. Under endoscope, we can identify the foramen of Monro, stria terminalis and the choroid plexus within the lateral ventricle, effectively avoid damage to the thalamostriate vein and superior choroidal vein, thereby fully exposing the tumor and reducing the risk of bleeding. More importantly, a multi-angle moving channel created by endoport is conducive to thoroughly removing tumors. In addition, navigating the endoport by a second surgeon, the endoscope can explore the occipital angle of the lateral ventricle and the third ventricle to remove the residual clot, thereby reducing the risk of postoperative fever or hydrocephalus. In the current study, the majority of patients with benign lesions and low-grade glioma obtained acceptable short-term and long-term postoperative outcomes, confirming the safety and effectiveness of the surgery procedure

It is noteworthy that all of the tumors in this study were mainly located in medial parts of the thalamus, adjacent to or protruding into the lateral ventricle and third ventricle. Previously, Rangel-Castilla and Spetzler classified the thalamus into six different regions based on the specific surgical approach and corridor [22]. Then, Oguz Baran et al described anatomic principles of approaches to the thalamus using a fiber dissection technique[1]. According to this surgical experience with cavernous malformations and cadaveric specimens, the surgical approaches for tumors located in the anterior and medial parts of the thalamus include supracarotid infrafrontal approach, transrostral translamina terminalis approach and anterior interhemispheric transcallosal approach. However, these surgical approaches still have corresponding risks besides the ordinary risks of small manipulation space, the damage of bridging 
veins, venous infarction, and retraction[11]. First, the supracarotid infrafrontal approach may injure the perforating arteries and lead to internal capsule stroke [26]; Second, the transrostral translamina terminalis approach needs to incise the rostrum and injury the minor forceps, lead to disconnection of frontal lobes[1]. Third, the anterior interhemispheric transcallosal approach requires severing midcallosal fibers, which may cause a severe syndrome of visual-verbal disconnection [19]. The choice of approach in our cases, unlike in these series, was the endoport-assisted endoscopic transcortical (middle frontal gyrus) transventricular approach. It provides a closer and broader exposure to the anatomical structure but minor damage to the brain parenchyma. The fact that no serious postoperative complications occurred in patients with benign lesions and low-grade glioma can reasonably verify this, although the incidence here is higher than $15 \%$ reported in previous studies $[23,24]$.

To deep-seated cerebral lesions, brain retraction is essential to maintain the corridor. Previously, this manipulation involved applying pressure to the overlying cerebral cortex via retractor blades. In some instances, it can cause neurological deficits, seizures, cognitive impairment, even leading to venous infarction $[16,17,28]$. Subsequently, Kelly et a/ proposed a less destructive method to expand the brain parenchyma by using a sequence of cylinders, the so-called"stereotactic tube-guided approach" [18]. Endoport-assisted surgery combined with the neuronavigation system is a modified form of them. The cylindrical sheath can distribute the retraction forces evenly and radially along with many points, thereby overcoming the high pressure on the nerve tissue caused by the limited area of the flat-bladed retractor[15]. The transparent sheath allows observing the surrounding tissues while preventing the bleeding in the corridor from affecting the visual field.

Furthermore, this technique ensures that the deep white matter tracts are split rather than transected [14]. As a working channel, the sheath provides a relatively large surgical corridor and allows for bimanual microsurgical instruments [17]. It also protects surrounding tissues from injuries caused by instruments being passed in and out of the corridor. In clinical practice, under the guidance of neuronavigation, the directed positioning of an access port can minimize cerebral retraction. Then, we fix the sheath with a pneumatic self-retaining holding device to free our hands to manipulate instruments. Sometimes, this technique is performed by two experienced surgeons, and both the endoscope and the endoport can be handheld to achieve a dynamic view with multi-angle views. Different from microscope surgery, the endoscope also provides a panoramic view through the inverted cone of light "flashlight effect" and can dynamically magnify it[20]. With the advantages of minimizing the required cortical corridor without sacrificing the intraoperative exposure, all patients with benign lesions and low-grade glioma achieved the results of GTR or NTR without seizures and severe neurological deficits.

Based on the clinical results in this study, we highlighted the following relevant experience. First of all, it should be noted that the postoperative outcome of thalamic glioblastoma is remains frustrating. We reasonably speculated that the 4 cases of high aggressiveness of glioblastoma lesions are not limited to the thalamus but also infiltrates the midbrain and even the pons, which is also a fundamental reason for the poor prognosis. That makes it challenging to achieve the goal of GTR by the surgery procedure alone. In addition, these patients had low preoperative KPS scores and apparent neurological deficits, and 
surgery cannot effectively improve the poor functional status. Most of the patients do not have the opportunity to receive adjuvant therapy, and the tumors relapse in a short period. In the future, modern MRI techniques integrating with diffusion tensor imaging, intraoperative MRI, and intraoperative neurophysiological monitoring will be applied better to define the tumor and the surrounding functional structures. Second, intraoperative tumor removal still relies on bimanual microsurgical techniques. Timely adjustment of the spatial position of surgical instruments and endoscope helps to avoid the spaceoccupying effect.

Moreover, skilled suction and dissection technique is the guarantee for the success of the operation. Third, continuous training and relevant anatomy knowledge should be taken to overcome the lack of stereoscopy of the endoscope [12]. Finally, this surgical approach has more advantages than supracarotid infrafrontal and transrostral translamina terminalis approaches for patients with obstructive hydrocephalus and dilated lateral ventricle.

The current study results should be interpreted with caution, as it only comprised a small number of patients from our center. First, to avoid the selection and treatment biases of the institution and its physicians, further studies from more centers are necessary to evaluate the optimal patient demographics, tumor characteristics, and long-term postoperative outcomes. Besides, we merely proposed an alternative surgical procedure for thalamic tumors located in the anterior and medial parts of the thalamus. However, it did not include a comparable control group of similar thalamic tumors treated with traditional microsurgery techniques. With the accumulation of experience in similar cases, the best indications for this surgical approach are expected to be further standardized

\section{Conclusions}

In conclusion, combining the advantages of neuronavigation, endoscopy, and endoport techniques, skilled micromanipulation can safely and effectively remove lesions located in the anterior and medial parts of the thalamus. With the advancement of the instruments, such as real-time navigation, threedimensional high definition endoscopy, and new material of the sheath tube, this surgical procedure may become prominent in deep-located tumor resection in the future.

\section{Declarations}

\section{Acknowledgements}

We sincerely acknowledge all the staff of department of neurosurgery, Zhongshan Hospital for helping perform the study and for the care of the study participants.

\section{Founding}

This work was supported in part by grants from the National Natural Science Foundation of China (81802893). 


\section{Conflict of interest}

All authors certify that they have no affiliations with or involvement in any organization or entity with any financial interest or non-financial interest in the subject matter or materials discussed in this manuscript.

\section{Availability of data and material}

The data that support the findings of this study are available from the corresponding author, upon reasonable request.

\section{Code availability}

Not applicable

\section{Ethics approval}

This study retrospectively evaluated patient data from our electronic medical records; Patient identities were anonymized before analysis. The entire study complied with the ethical guidelines of the 1975 Declaration of Helsinki (as revised in Brazil in 2013). The Zhongshan Hospital Research Ethics Committee approved the study protocol. Director's consent for publication was granted as well.

\section{Consent to participate}

All participants, or their next of kin, when applicable, provided informed consent before surgery.

\section{Consent for publication}

All authors declare that they approve and consent to this submission by the corresponding author and support its publication, if accepted. All authors have read and approved the manuscript.

\section{Author contribution}

Conception and design: Xiaobiao Zhang. Acquisition of data: Yeh Yu Yang, Chen Li, Tengfei Liu. Analysis and interpretation of data: Shuang Liu, Tao Xie. Drafting the article: Shuang Liu, Tao Xie. Critically reviewing the article: Chongjing Sun, Silin Wu, Liangliang Yang, Zeyang Li, Fan Hu. Reviewed submitted version of the manuscript: all authors. Approved the final version of the manuscript on behalf of all authors: Xiaobiao Zhang. Administrative/technical/material support: all authors. Agreement to be accountable for all aspects of work: Shuang Liu, Xiaobiao Zhang..

\section{References}

1. Baran O, Baydin S, Gungor A, Balak N, Middlebrooks E, Saygi T, et al. (2019) Surgical Approaches to the Thalamus in Relation to the White Matter Tracts of the Cerebrum. World neurosurgery 128:e1048e1086. https://doi.org/10.1016/j.wneu.2019.05.068. 
2. Baroncini M, Vinchon M, Minéo JF, Pichon F, Francke JP, Dhellemmes P (2007) Surgical resection of thalamic tumors in children: approaches and clinical results. Child's nervous system : ChNS : official journal of the International Society for Pediatric Neurosurgery 23(7):753-

60. https://doi.org/10.1007/s00381-007-0299-4.

3. Beks JW, Bouma GJ, Journée HL (1987) Tumours of the thalamic region. A retrospective study of 27 cases. Acta neurochirurgica 85(3-4):125-7. https://doi.org/10.1007/bf01456108.

4. Bosnjak R, Antolin A, Jeglic A, Felbabic T, Velnar T (2020) Navigated Endoport in the Purely Endoscopic Microsurgery of Intraventricular and Other Deep-Seated Brain Lesions: A Case Report. Acta medica academica 49 Suppl 1:70-77. https://doi.org/10.5644/ama2006-124.309.

5. Broadway SJ, Ogg RJ, Scoggins MA, Sanford R, Patay Z, Boop FA (2011) Surgical management of tumors producing the thalamopeduncular syndrome of childhood. Journal of neurosurgery Pediatrics 7(6):589-95. https://doi.org/10.3171/2011.4.peds119.

6. Cao L, Li C, Zhang Y, Gui S (2015) Surgical resection of unilateral thalamic tumors in adults: approaches and outcomes. BMC neurology 15:229. https://doi.org/10.1186/s12883-015-0487-x.

7. Cinalli G, Aguirre DT, Mirone G, Ruggiero C, Cascone D, Quaglietta L, et al. (2018) Surgical treatment of thalamic tumors in children. Journal of neurosurgery Pediatrics 21(3):247257. https://doi.org/10.3171/2017.7.peds16463.

8. Cuccia V, Monges J (1997) Thalamic tumors in children. Child's nervous system : ChNS : official journal of the International Society for Pediatric Neurosurgery 13(10):514-20; discussion 521. https://doi.org/10.1007/s003810050128.

9. Darbar A, Mustansir F, Hani U, Sajid MI (2020) A Review of Common Endoscopic Intracranial Approaches. Asian journal of neurosurgery 15(3):471-478. https://doi.org/10.4103/ajns.AJNS_367_19.

10. Ding D, Starke RM, Crowley RW, Liu KC (2015) Endoport-assisted microsurgical resection of cerebral cavernous malformations. Journal of clinical neuroscience : official journal of the Neurosurgical Society of Australasia 22(6):1025-9. https://doi.org/10.1016/j.jocn.2015.01.004.

11. Elshamy W, Burkard J, Gerges M, Erginoglu U, Aycan A, Ozaydin B, et al. (2021) Surgical approaches for resection of third ventricle colloid cysts: meta-analysis. Neurosurgical review https://doi.org/10.1007/s10143-021-01486-5.

12. Esposito F, Cappabianca P (2013) Neuroendoscopy: General Aspects and Principles. World neurosurgery $79(2$, Supplement):S14.e7-

S14.e9. https://doi.org/https://doi.org/10.1016/j.wneu.2012.02.033.

13. Gao H, Liu C, Zhang Y (2018) Neuro-endoscope for skull base tumors. Clinical neurology and neurosurgery 170:102-105. https://doi.org/10.1016/j.clineuro.2018.05.009. 
14. Greenfield JP, Cobb WS, Tsouris AJ, Schwartz TH (2008) Stereotactic minimally invasive tubular retractor system for deep brain lesions. Neurosurgery 63(4 Suppl 2):334-9; discussion 33940. https://doi.org/10.1227/01.neu.0000334741.61745.72.

15. Harris AE, Hadjipanayis CG, Lunsford LD, Lunsford AK, Kassam AB (2008) Microsurgical removal of intraventricular lesions using endoscopic visualization and stereotactic guidance. Neurosurgery 62 Suppl 2:622-9. https://doi.org/10.1227/01.neu.0000316266.97714.da.

16. Hongo K, Kobayashi S, Yokoh A, Sugita K (1987) Monitoring retraction pressure on the brain. An experimental and clinical study. Journal of neurosurgery 66(2):270-

5. https://doi.org/10.3171/jns.1987.66.2.0270.

17. Jo KW, Shin HJ, Nam DH, Lee Jl, Park K, Kim JH, et al. (2011) Efficacy of endoport-guided endoscopic resection for deep-seated brain lesions. Neurosurgical review 34(4):457-

63. https://doi.org/10.1007/s10143-011-0319-4.

18. Kelly PJ, Kall BA, Goerss SJ (1987) Computer-interactive stereotactic resection of deep-seated and centrally located intraaxial brain lesions. Applied neurophysiology 50(1-6):107-

13. https://doi.org/10.1159/000100693.

19. Levin HS, Rose JE (1979) Alexia without agraphia in a musician after transcallosal removal of a left intraventricular meningioma. Neurosurgery 4(2):168-74. https://doi.org/10.1227/00006123197902000-00011.

20. McLaughlin N, Prevedello DM, Engh J, Kelly DF, Kassam AB (2013) Endoneurosurgical resection of intraventricular and intraparenchymal lesions using the port technique. World neurosurgery 79(2 Suppl):S18.e1-8. https://doi.org/10.1016/j.wneu.2012.02.022.

21. Puget S, Crimmins DW, Garnett MR, Grill J, Oliveira R, Boddaert N, et al. (2007) Thalamic tumors in children: a reappraisal. Journal of neurosurgery 106(5 Suppl):354-

62. https://doi.org/10.3171/ped.2007.106.5.354.

22. Rangel-Castilla L, Spetzler RF (2015) The 6 thalamic regions: surgical approaches to thalamic cavernous malformations, operative results, and clinical outcomes. Journal of neurosurgery 123(3):67685. https://doi.org/10.3171/2014.11.Jns14381.

23. Sabanci PA, Aras Y, Ali A, Unal TC, Dolen D, Sencer S, et al. (2017) Transcortical Removal of Third Ventricular Colloid Cysts: Comparison of Conventional, Guided Microsurgical and Endoscopic Approaches and Review of the Literature. Turkish neurosurgery 27(4):546-

557. https://doi.org/10.5137/1019-5149.Jtn.17142-16.2.

24. Stachura K, Libionka W, Moskała M, Krupa M, Polak J (2009) Colloid cysts of the third ventricle. Endoscopic and open microsurgical management. Neurologia i neurochirurgia polska 43(3):251-7. 
25. Tovi D, Schisano G, Liljeqvist B (1961) Primary tumors of the region of the thalamus. Journal of neurosurgery 18:730-40. https://doi.org/10.3171/jns.1961.18.6.0730.

26. Waldron JS, Lawton MT (2009) The supracarotid-infrafrontal approach: surgical technique and clinical application to cavernous malformations in the anteroinferior Basal Ganglia. Neurosurgery 64(3 Suppl):ons86-95; discussion ons95. https://doi.org/10.1227/01.Neu.0000335647.71014.07.

27. Wong TT, Chen HH, Liang ML, Hsieh KL, Yang YS, Ho DM, et al. (2016) Clinical considerations and surgical approaches for low-grade gliomas in deep hemispheric locations: thalamic lesions. Child's nervous system : ChNS : official journal of the International Society for Pediatric Neurosurgery 32(10):1895-906. https://doi.org/10.1007/s00381-016-3148-5.

28. Yokoh A, Sugita K, Kobayashi S (1983) Intermittent versus continuous brain retraction. An experimental study. Journal of neurosurgery 58(6):918-23. https://doi.org/10.3171/jns.1983.58.6.0918.

\section{Tables}

Table 1. Baseline characteristics of study subjects 


\begin{tabular}{|ll|}
\hline & N (\%), median (range) \\
\hline Age, years & $55 \rrbracket 13,63 \rrbracket$ \\
\hline Sex, male & $7(58.3)$ \\
\hline Clinical symptoms & \\
\hline Headache and/or dizziness & $7(58.3)$ \\
\hline hemiparesis & $3(25.0)$ \\
\hline Others & $2(16.7)$ \\
\hline KPS score at admission & $80(50,90)$ \\
\hline Treatment before surgery & \\
\hline None & $7(58.3)$ \\
\hline Surgical treatment & $2(16.7)$ \\
\hline Medical treatment & $3(25.0)$ \\
\hline Time from onset to operation, weeks & $4(1,24)$ \\
\hline Tumor size, mm & $29(16,56)$ \\
\hline Tumor location & \\
\hline Region 2 & $1(8.3)$ \\
\hline Region 1,2 & $5(41.7)$ \\
\hline Region 2,4 & $1(8.3)$ \\
\hline Region 1,2,4 & $5(41.7)$ \\
\hline Side of surgery left & $5(41.7)$ \\
\hline Tumor pathology & \\
\hline Cavernous malformation & $3(25.3)$ \\
\hline Pilocytic astrocytoma (WHO I) & \\
\hline Low grade glioma (WHO II) & \\
\hline Glioblastoma (WHO IV) & \\
\hline
\end{tabular}

Continuous variables are presented as median (range), categorical variables as $\mathrm{N}(\%)$. Abbreviations: KPS, Karnofsky performance status; WHO, world health organization Table 2. The extent of resection and clinical outcome of study subjects 


\begin{tabular}{|lll|}
\hline & Benign lesions and low grade gliomas $(\mathbf{N}=8)$ & $\begin{array}{l}\text { Glioblastoma } \\
(\mathbf{N}=4)\end{array}$ \\
\hline Resection results & & $0(0)$ \\
\hline GTR & $8(100)$ & $3(75)$ \\
\hline NTR & $0(0)$ & $1(25)$ \\
\hline STR & $0(0)$ & \\
\hline KPS score at discharge & & $1(25)$ \\
\hline No change & $2(25)$ & $0(0)$ \\
\hline Improved & $4(50)$ & $3(75)$ \\
\hline Deteriorated & $2(25)$ & $0(0)$ \\
\hline Clinical symptoms & & $1(25)$ \\
\hline Relieved & $7(87.5)$ & $3(75)$ \\
\hline Unrelieved & $0(0)$ & $2(50)$ \\
\hline New onset & $1(12.5)$ & $0(0)$ \\
\hline Postoperative complications & & $3(75)$ \\
\hline Present & $3(37.5)$ & $1(25)$ \\
\hline Absent & $5(62.5)$ & \\
\hline Adjuvant treatment & $4(50)$ & $2(50)$ \\
\hline Yes & $2(25)$ & \\
\hline No & & \\
\hline Intraoperative ostomy & & \\
\hline NICU days & & \\
\hline & & \\
\hline
\end{tabular}

Continuous variables are presented as median (range), categorical variables as $\mathrm{N}(\%)$.

Abbreviations: GTR, gross-total resection; NTR, near-total resection; STR, subtotal resection; KPS, Karnofsky performance status; NICU, neurosurgical intensive care unit.

\section{Figures}




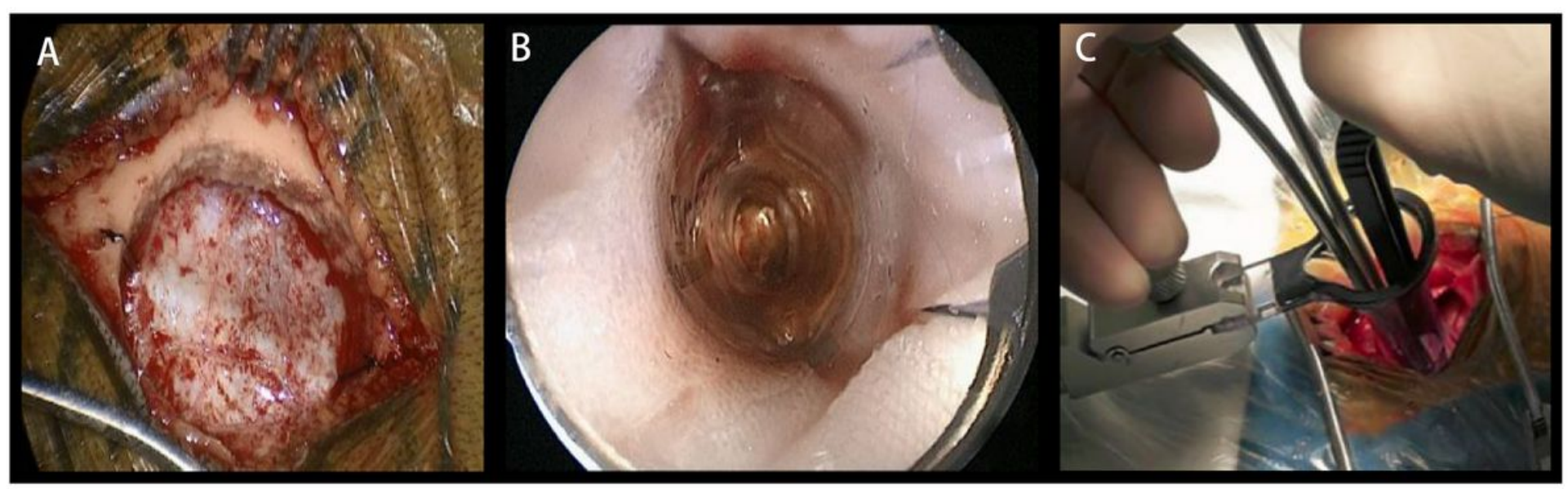

\section{Figure 1}

Surgical procedure A. After a straight incision about $5 \mathrm{~cm}$ long located anterior to the middle frontal gyrus, the circular craniotomy bone flap with approximately $3 \mathrm{~cm}$ in diameter was made. B. Choosing the appropriate endoport system to expand the cortex and advance along the planned trajectory until the lateral ventricle. C. The positional relationship among the endoscope, the bipolar electrocoagulator, and the suction tube during operation

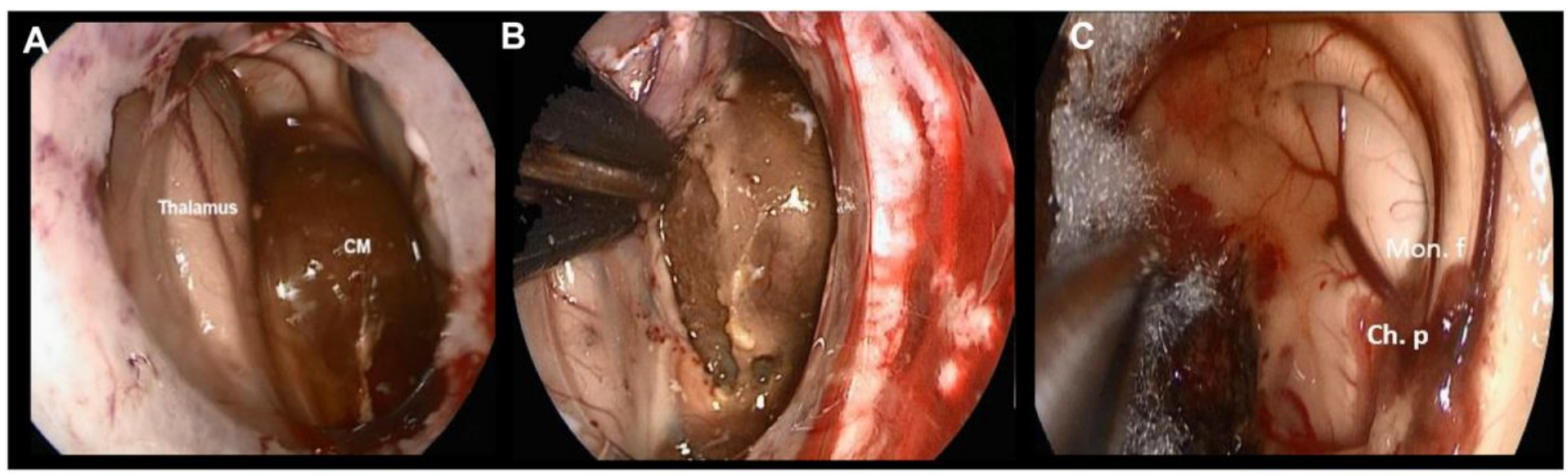

\section{Figure 2}

Gross total resection of cavernous malformation A. Panoramic view of cavernous malformation (CM) after the endoscope entered the ventricle. B. Completely separated the boundary of CM from thalamus. C. The CM was removed entirely and the choroid plexus and foramen of Monro were exposed. 


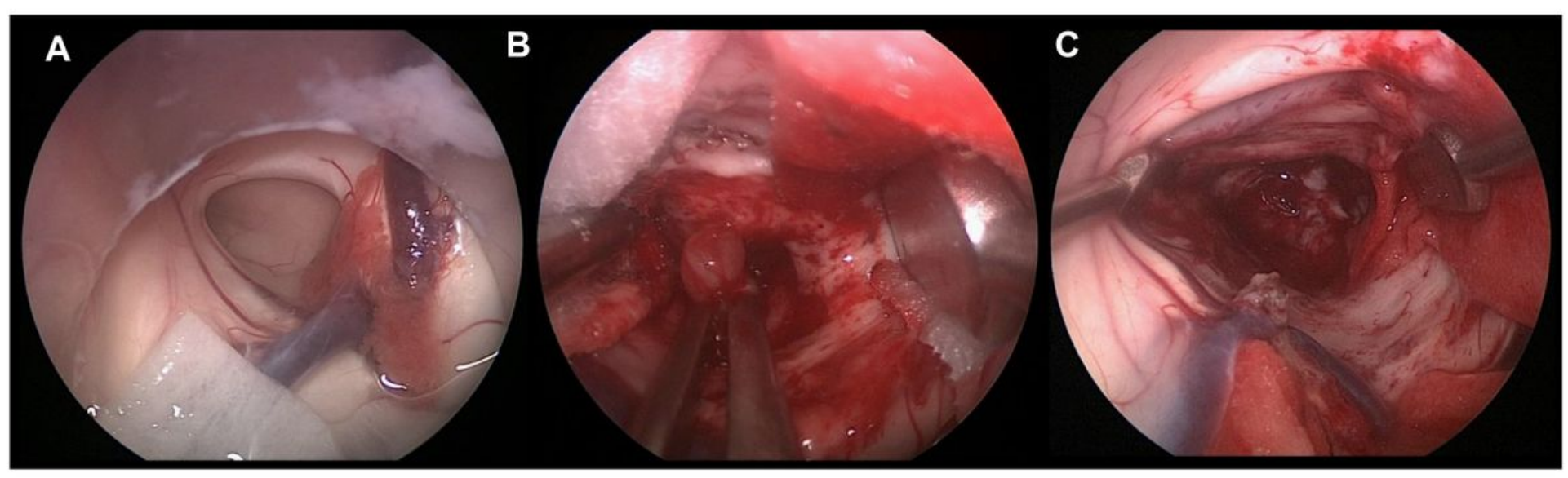

\section{Figure 3}

Near-total resection of glioblastoma A. Endoscopic panoramic view of lateral ventricular. B. Intratumoral decompression followed by piecemeal total resection was adopted under high magnification. $\mathrm{C}$. The CM was removed entirely and the choroid plexus and foramen of Monro were visualized clearly.

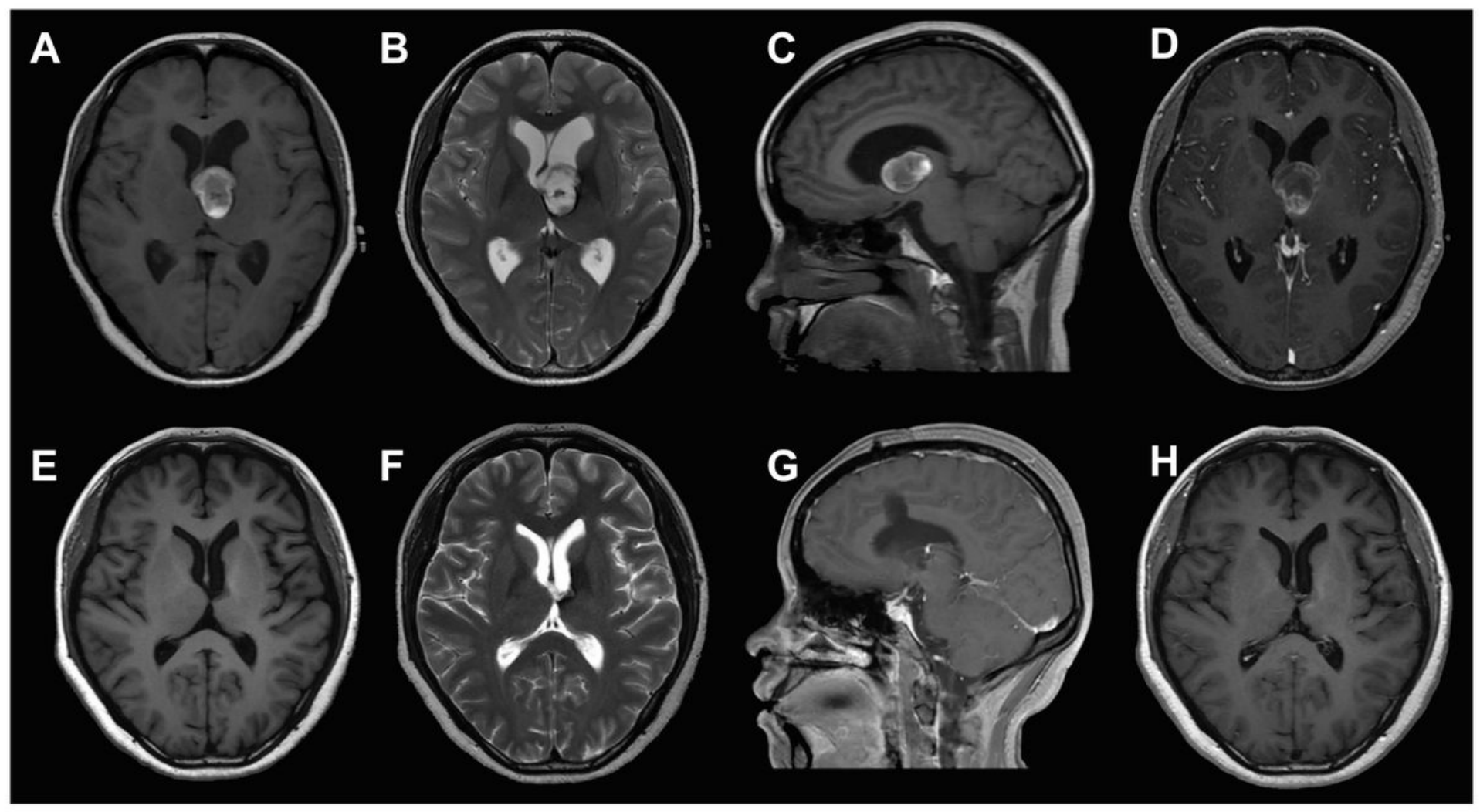

\section{Figure 4}

Preoperative and postoperative magnetic resonance imaging (MRI) of the patient with thalamus cavernous malformation Preoperative MRI sequence A. Axial T1-weighted; B. Axial T2-weighted; C. Sagittal T1-weighted; D. Axial T1-weighted with contrast. Postoperative MRI sequence E. Axial T1weighted; F. Axial T2-weighted; G. Sagittal T1-weighted with contrast; H. Axial T1-weighted with contrast. 


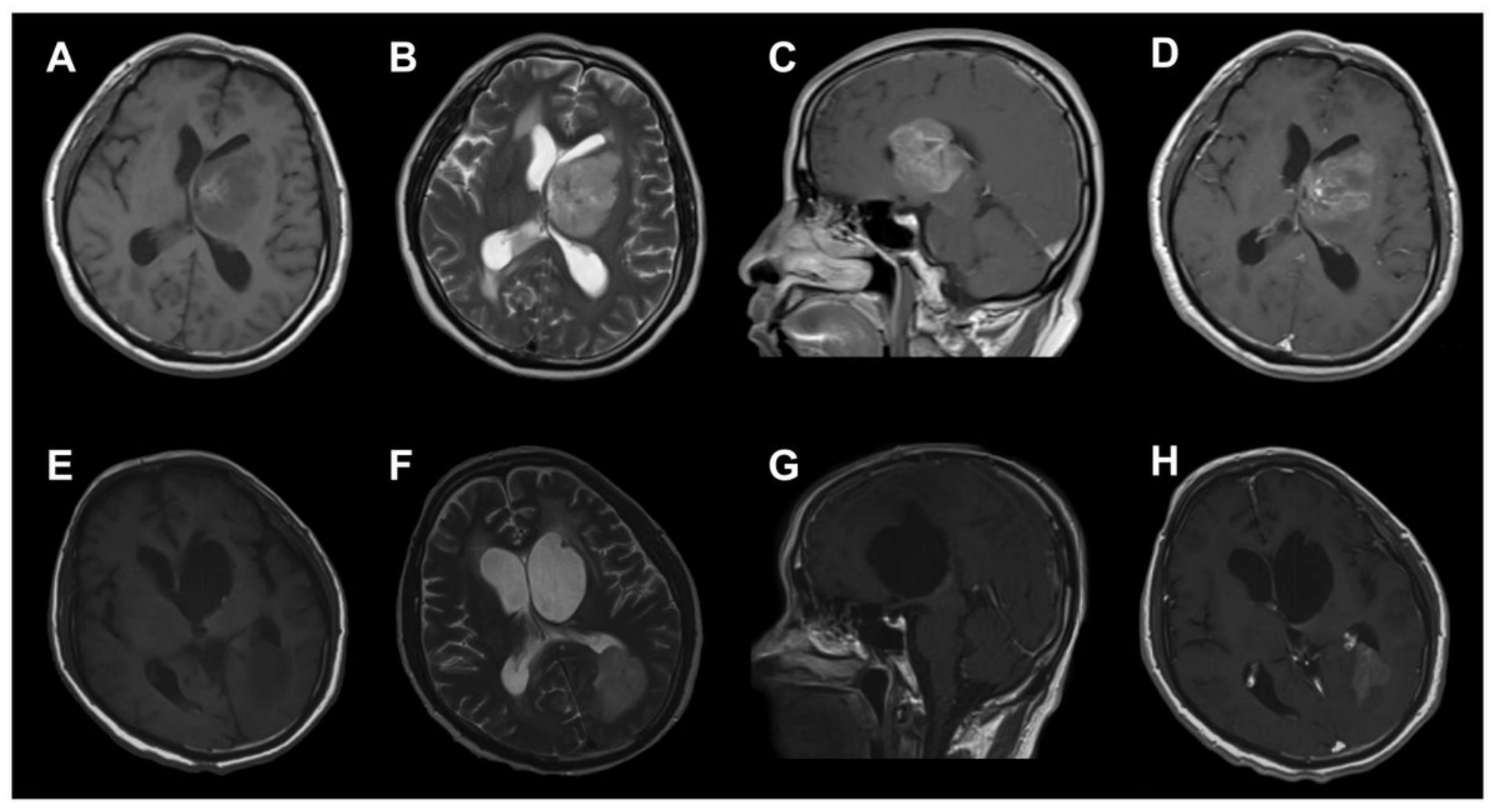

\section{Figure 5}

Preoperative and postoperative magnetic resonance imaging (MRI) of the patient with thalamus glioblastoma Preoperative MRI sequence: A. Axial T1-weighted; B. Axial T2-weighted; C. Sagittal T1weighted with contrast; D. Axial T1-weighted with contrast. Postoperative MRI sequence: E. Axial T1weighted; F. Axial T2-weighted; G. Sagittal T1-weightedwith contrast; H. Axial T1-weighted with contrast showed signs of tumor metastasis. 